17 months. Thematic analysis yielded the identification and categorization of major factors associated with implementation of the delirium identification process. Factors identified inform barriers as well as facilitators: 1) patient factors (affective, cognitive and physical status, coping mechanisms, social support);2) provider factors (competing demands, skills); 3) structural factors (environmental adaptations for age-related changes, comfort, privacy); 4) organizational-level factors (staffing, morale); and 5) protocol innovation (perceived utility, efficiency). Clinicians described the protocol as efficient and having potential for integration into daily routines. As one of the first studies to test a 2-step process for delirium identification and to involve NAs, our findings have role implications and describe the need for a multi-faceted, systemic approach to implementation.

\section{IMPLICATIONS OF CONCURRENT LOW MUSCLE AREA IN DETERMINING THE SIGNIFICANCE OF INTER-MUSCULAR FAT IN OLD ADULTS}

S. Farsijani ${ }^{1}$, P. Cawthon ${ }^{2}$, A. Santanasto ${ }^{1}$, B. Goodpaster ${ }^{3}$, S. Kritchevsky ${ }^{4}$, R. Boudreau ${ }^{5}$, T. Harris 6 , A. Newman ${ }^{1}, 1$. University of Pittsburgh, 2. San Francisco Coordinating Center, California Pacific Medical Center Research Institute and Associate Adjunct Professor Department of Epidemiology and Biostatistics University of California, San Francisco, 3. Translational Research Institute for Metabolism and Diabetes, 4. Wake Forest School of Medicine, 5. Dept of Epidemiology, Univ of Pittsburgh, 6. National Institute on Aging

Fat "infiltration" in skeletal muscle is considered an important predictor of impaired muscle function in older adults. Muscle fat depots is seen in broad pathological conditions, from obesity (in which relatively preserved muscle tissue is "infiltrated" by fat) to cancer cachexia (in which muscle wasting is concurrent with fat "replacement" of the muscle). However, whether the importance of muscle fat depots varies by the amount of muscle present is unknown.

Objective: In older adults, to determine whether the associations between mid-thigh inter-muscular fat area and anthropometric characteristics, physical function and health conditions vary by low or high mid-thigh muscle cross-sectional area.

Design: Participants $(n=1903$; age $74-85 y ; 52.2 \%$ women) from the Health ABC study, were categorized into four groups; high inter-muscular-fat with low vs. high muscle area $\left(\mathrm{fat}^{\wedge} \mathrm{H} / \mathrm{muscle}^{\wedge} \mathrm{L}\right.$ and $\mathrm{fat}{ }^{\wedge} \mathrm{H} /$ muscle $\left.^{\wedge} \mathrm{H}\right)$ and low intermuscular-fat with low vs. high muscle area $\left(\mathrm{fat}^{\wedge} \mathrm{L} / \mathrm{muscle}^{\wedge} \mathrm{L}\right.$ and $\left.\mathrm{fat}^{\wedge} \mathrm{L} / \mathrm{muscle}^{\wedge} \mathrm{H}\right)$ based on 2003-04 CT data. Body composition (DXA), physical function and metabolic profile were compared across groups.

Results: Compared to $\mathrm{fat}^{\wedge} \mathrm{H} / \mathrm{muscle}^{\wedge} \mathrm{H}$ group, individuals in $\mathrm{fat}^{\wedge} \mathrm{H} /$ muscle $^{\wedge} \mathrm{L}$ had lower BMI, appendicular lean mass (aLM) and fat mass. They had experienced an accelerated aLM loss over the previous 5-yrs. In the presence of low muscle area, physical function outcomes were mainly determined by the muscle area, and not the inter-muscular fat. Fat ${ }^{\wedge} \mathrm{H} /$ muscle ${ }^{\wedge} \mathrm{H}$ group had a metabolic profile resembling metabolic syndrome; lower HDL, hyperglycemia (in women), and hypertriglyceridemia (in men) compared to fat $^{\wedge} \mathrm{H} /$ muscle $^{\wedge} \mathrm{L}$ group.
Conclusions: The negative effects of inter-muscular fat in aging are greatly influenced by the presence of concurrent low muscle mass, sex and age.

\section{INCIDENCE OF DEMENTIA IN ADULTS WITH CEREBRAL PALSY: A UK COHORT STUDY}

K. Smith ${ }^{1}$, M. Peterson ${ }^{2}$, C. Victor ${ }^{3}, \mathrm{~J}_{\text {. Ryan }}{ }^{4}, 1$. University of Surrey, 2. University of Michigan, 3. Brunel University London, 4. Royal College of Surgeons in Ireland

Researchers have previously hypothesised that ageing with cerebral palsy (CP) may be associated with an increased risk of developing dementia. However no study has yet formally investigated this proposition while accounting for important morbidities such as intellectual disabilities (ID) or epilepsy. We used a United Kingdom primary care database as our data source (CPRD Gold). Data for 1,705 adults with CP (age range 18-89) and 5,115 age, sex and general practice matched controls without $\mathrm{CP}$ was extracted from the database. Dementia was defined by read codes. We used stratified Cox Proportional Hazards Regression analyses to determine incidence of dementia. In total there were 51 cases of incident dementia over follow-up. There was no significant difference in the risk of developing dementia between people with CP and those without CP (HR 1.40, 95\% CI 0.58-3.40). We then repeated our analysis comparing those with CP and either ID or epilepsy with adults without CP. Those with ID co-morbidity had a higher risk of developing dementia than people with no CP (HR 7.11, 95\% CI 1.3836.75 ) as did those with CP and epilepsy (HR 12.0, 95\% CI 1.34-107.36). Results suggest that only those people with CP and ID or epilepsy are more likely to develop dementia. This suggests that the previously speculated link between CP and dementia may be due to other neurologic or intellectual co-morbidities rather than as a direct effect of CP; however, more work is needed to confirm this link and the mechanisms underlying it.

\section{INCIDENCE OF LOWER-EXTREMITY FRACTURES IN U.S. NURSING HOMES}

K. Sine ${ }^{1}$, Y. Lee ${ }^{2}$, A. Zullo ${ }^{3}$, L. Daiello ${ }^{4}$, T. Zhang ${ }^{3}$, S. Berry ${ }^{5}$, 1. Hebrew SeniorLife, Institute for Aging Research, 2.

Brown University, 3. Brown University School of Public Health, 4. Brown University, School of Public Health, 5. Hebrew SeniorLife

Limited studies suggest lower extremity (LE) fractures are morbid events for nursing home $(\mathrm{NH})$ residents. Our objective was to conduct a nationwide study comparing the incidence and potential risk factors associated with hip versus non-hip LE (femoral shaft and tibia-fibula) fractures in the NH. We included all U.S. long-stay residents aged $\geq 65$ years enrolled in Medicare 1/1/2008-12/31/2009 ( $\mathrm{N}=1,257,279)$. Residents were followed from long-stay qualification until the first event of LE fracture, death, or end of follow-up (2-years). Fractures were classified using Medicare diagnostic and procedural codes. Function, cognition, and medical status were obtained from the Minimum Data Set prior to long-stay qualification. Incidence rates (IR) were calculated as the total number of fractures divided by person-years.

During 42,800 person-years follow-up, 52,177 residents had a LE fracture (43,695 hip, 6,001 femoral shaft, 2,481 tibia-fibula). The unadjusted IR of LE fractures were 
1.32/1,000 person-years (95\% CI, 1.27-1.38) for tibiafibula, 3.20/1,000 person-years (95\% CI, 3.12-3.29) for femoral shaft, and 23.32/1,000 person years $(95 \% \mathrm{CI}$, 23.11-23.54) for hip. As compared with hip fracture residents, non-hip LE fracture residents were more likely to be immobile (58.1\% vs $18.4 \%$ ), dependent in all ADLs $(31.6 \%$ vs $10.8 \%)$, transferred mechanically $(20.5 \%$ vs $4.4 \%)$, overweight (mean BMI $26.6 \mathrm{~kg} / \mathrm{m} 2$ vs. 24.0), and have diabetes $(34.8 \%$ vs $25.7 \%)$. Our findings that non-hip LE fractures often occur in severely functionally impaired residents suggests these fractures may occur during lifting and transferring. The resident differences in our study highlight the need for distinct prevention strategies for hip and non-hip LE fractures.

\section{INEQUALITY ACROSS NURSING HOMES: MEASUREMENT OF RACIAL SEGREGATION AMONG NURSING HOMES IN THE UNITED STATES}

D. Mack, K. Lapane, University of Massachusetts Medical School

Nursing homes are not immune to the residual effects of lawful racial segregation that was previously instituted in the United States. However, a standardized approach to measure segregation in nursing homes does not appear to be established. A systematic review was conducted to identify all formal measurement approaches to evaluate racial segregation among nursing home facilities, and to then identify facility-level associations between segregation and quality of care. PubMed, Scopus and Web of Science databases were searched for studies relating to segregation among nursing homes across the United States. Following the PRISMA guidelines, literature was systematically evaluated and studies were excluded if articles were not in English, were not original, or did not formally measure racial segregation of nursing homes residents with regional-level data. Formal segregation measures identified included the Dissimilarity Index, the Disparities Quality Index, the Modified Thiel's Entropy Index, the Gini coefficient, and adapted models. The most common data sources used were the Minimum Data Set (MDS; resident-level data), the Certification and Survey Provider Enhanced Reporting data (CASPER; facility-level data), and the Area Resource File/ US Census Data (regionallevel data). Overall, the measurement of racial segregation among nursing homes is heterogeneous. While there are limitations to each methodology, this review can be used as a reference when trying to determine the best approach to measure racial segregation in future studies. Moreover, most studies showed evidence of racial segregation among nursing home facilities and subsequently, a negative impact of segregation on racial minorities and facility quality outcomes.

\section{INVOLUNTARY TREATMENT AMONG OLDER ADULTS WITH COGNITIVE IMPAIRMENT RECEIVING NURSING CARE AT HOME.}

V. Moermans ${ }^{1}$, M. Bleijlevens ${ }^{2}$, H. Verbeek ${ }^{3}$, F. Tan ${ }^{4}$, K. Milisen, J. Hamers ${ }^{6}$, 1. RN, MSc, 2. Department of Health Services Research, Care and Public Health Research Institute (CAPHRI), Maastricht University, Maastricht, Limburg, the Netherlands, 3. Department of Health Service Research, Care and Public Health Research Institute (CAPHRI), Maastricht University, Netherlands,
4. Departement Methodoloqy and Statistics, Care and Public Health Research Institute (CAPHRI), Maastricht University, The Netherlands, 54Department of Public Health and Primary Care, Accent VV, KU Leuven, Leuven, Belgium; and Department of Internal Medicine, Division of Geriatric Medicine, University Hospitals Leuven, Leuven, Belgium, 6. Department of Health Services Research, Care and Public Health Research Institute, Maastricht University, Netherlands

The application of involuntary treatment, including the use of physical restraints (e.g. belts), psychotropic drugs (e.g. inappropriate use of anti-psychotics) and non-consensual care (e.g. restricting transportation), has a negative impact on the quality of life of cognitive impaired older adults living at home and their caregivers. Research on this topic is scarce. The aim of this study is to gain insight into involuntary treatment usage among older adults with cognitive impairment receiving home nursing. This cross-sectional study, analysed data from 1194 randomly selected older adults with cognitive impairments receiving nursing care at home in Belgium. A district nurse filled out a questionnaire based on data available in patient records. The application of involuntary treatment, who request and apply it, and associated factors were assessed. Data were analysed using descriptive analyses and random intercept logistic regression. Involuntary treatment was used in $52 \%$ of the total sample. The use of involuntary treatment was associated with ADL-dependency (O.R. 1.5), cognitive impairment (O.R. 1.39) and informal caregiver burden (O.R. 1.05). Informal caregivers most often requested the use of involuntary treatment and nurses most often applied it. This study confirms that involuntary treatment is common in home nursing and a multifactorial approach (including policy, education, consultation and alternatives) is needed to prevent and reduce it. Whenever caregivers have the skills and knowledge about alternative methods, there is no reason to apply care that older adults resist to. The insights gained through this study, will help researchers and professional caregivers to develop such a multifactorial approach.

\section{ISOLATED SYSTOLIC HYPERTENSION AND RISKS OF STROKE IN THE OLDEST OLD}

R. Kheirbek ${ }^{1}$, A. Fokar ${ }^{2}$, 1. George Washington University School of Medicine and Health Sciences, 2. Washington DC VA Medical Center

Aim With nearly 800,000 Americans suffering from stroke each year, it became a leading cause of death. Hypertension is the chief culprit, yet optimal blood pressure for the oldest old remains controversial. We investigated the association hypertension and stroke risk in an oldest old. Methods The study included 86,892 community-dwelling veterans born between 1910 - 1915 survived to at least age 80. Retrospective longitudinal cohort study. Data from the Department of Veterans Affairs (VA) Informatics and Computing Infrastructure were used. Kaplan-Meier method estimated cumulative incidence of stroke in the two groups (those with hypertension and those without hypertension) and compared using the logrank test. Cox proportional hazards models were used to estimate unadjusted hazard ratios. We estimated hypertension onset age by calculating the time difference between date of diagnosis and date of birth Results Ninety-seven percent 